\title{
Consumption of meals prepared away from home is associated with inadequacy of dietary fiber, vitamin $C$ and mineral intake among Japanese adults: analysis from the 2015 National Health and Nutrition Survey
}

Mai Matsumoto, Aki Saito, Chika Okada, Emiko Okada, Ryoko Tajima and Hidemi Takimoto* (D)

\begin{abstract}
Background: Consumption of home-prepared meals may lead to better nutritional intake. Few studies have examined the association between the frequency of consuming meals prepared away from home and the inadequacy of nutritional intake based on nutritional standards and food group intake. We therefore aimed to investigate this issue among Japanese adults.
\end{abstract}

Methods: This study was a secondary analysis of the 2015 National Health and Nutrition Survey in Japan, involving 921 men and 1300 women aged 18-64 years, conducted as a cross-sectional survey. The frequency of consuming meals prepared away from home was defined using two questions inquiring about the frequency of eating out and take-away meals. Data on dietary intake were collected using a one-day semi-weighed household dietary record. Participants were stratified into three groups based on the frequency of consuming meals prepared away from home (High, Moderate, Low). The inadequacy of each nutrient intake was assessed by comparing estimated average requirement (EAR) level for 14 nutrients and the range of the dietary goal (DG) for seven nutrients according to the 2015 version of the Dietary Reference Intakes for Japanese. Group differences in nutrients adequacy were assessed using the covariate and logistic regression analysis. Food intake was also compared across the groups by classifying each food item into 17 groups based on Standard Tables of Food Composition.

Results: The proportion of participants who consumed home-prepared meals almost every day were $34.9 \%$ among men and $46.8 \%$ among women, and the proportion of those consuming a higher frequency of meals prepared away from home were 14.7 and 6.3\%, respectively. A higher frequency of consuming meals prepared away from home was associated with inadequacy of dietary fiber, vitamin $\mathrm{C}$ and minerals (iron, magnesium and potassium) intake, and with lower intake of vegetables and higher intake of fat and oils.

* Correspondence: thidemi@nibiohn.go.jp

Department of Nutritional Epidemiology and Shokuiku, National Institutes of Biomedical Innovation, Health, and Nutrition, 1-23-1 Toyama, Shinjuku-ku,

Tokyo 162-8636, Japan

(c) The Author(s). 2021 Open Access This article is licensed under a Creative Commons Attribution 4.0 International License, which permits use, sharing, adaptation, distribution and reproduction in any medium or format, as long as you give appropriate credit to the original author(s) and the source, provide a link to the Creative Commons licence, and indicate if changes were made. The images or other third party material in this article are included in the article's Creative Commons licence, unless indicated otherwise in a credit line to the material. If material is not included in the article's Creative Commons licence and your intended use is not permitted by statutory regulation or exceeds the permitted use, you will need to obtain permission directly from the copyright holder. To view a copy of this licence, visit http://creativecommons.org/licenses/by/4.0/. The Creative Commons Public Domain Dedication waiver (http://creativecommons.org/publicdomain/zero/1.0/) applies to the data made available in this article, unless otherwise stated in a credit line to the data. 


\begin{abstract}
(Continued from previous page)
Conclusions: High frequency of consuming meals prepared away from home was associated with insufficient intake of dietary fiber, vitamin C and multiple minerals among Japanese adults. These nutrients may be the potential target of interventions aimed at improving nutrient intake in individuals who predominantly eat food prepared away from home.
\end{abstract}

Keywords: Meal prepared away from home, Nutrition inadequacy, Mineral, Japanese, NHNS

\section{Background}

The frequency of consuming meals prepared away from home has been reported to influence the quality of dietary intake [1]. Consuming meals prepared away from home is associated with higher intake of energy, fat, and sodium, and with lower intake of dietary fiber, vitamin $\mathrm{C}$, and several minerals such as iron and calcium [2-13]. Additionally, eating meals prepared away from home has been linked to reduced consumption of healthy foods such as vegetables, fruit and dairy products [14-18]. Moreover, increased frequency of eating out and takeaway meals has been associated with an increase in body weight, body mass index (BMI), and waist circumference $[14,18-20]$, and increased risk of obesity [21-23], insulin resistance, diabetes mellitus [16, 23, 24], and depression $[9,25,26]$. These findings suggest that a higher frequency of eating meals prepared away from home can affect not only the quality of diet, but also physical health.

A shift from food prepared at home to convenient/ easy-to-prepare food and food prepared outside the home has been reported in the United States [27]. Additionally, high frequency of consumption of food prepared away from home has been reported in other highincome countries such as United Kingdom, and Japan $[18,28]$. As the frequency of consumption of food prepared away from home increases, studies have examined the association between the frequency of consuming meals prepared away from home and intakes of energy and specific nutrients such as fat, sodium, vitamin C, iron, and calcium [2-13]. However, there are only a small number of studies that compared the nutritional adequacy of the subjects' dietary intakes, according to their frequency of consuming meals prepared away from home [18]. Thus, it is crucial to examine the association between frequency of consuming meals prepared away from home and overall dietary quality in order to identify more effective public health nutritional intervention. In addition, it would be helpful to assess food group intake according to the frequency of meals prepared away from home in order to understand its relationship with the nutrient intake adequacy. Therefore, we aimed to examine the association of the frequency of consuming meals prepared away from home with nutrient intake inadequacy and food group intake among Japanese adults aged 18-64 years using data from the 2015 National Health and Nutrition Survey in Japan (NHNS).

\section{Methods}

Data source and study population

The NHNS is a nationally representative cross-sectional annual survey conducted by local public health centers under the supervision of the Ministry of Health, Labour, and Welfare. The present study was based on data from the 2015 NHNS conducted between November 1 to 30, 2015. Details of the 2015 NHNS has been described elsewhere $[29,30]$. Briefly, the participants, who included households and family members (aged $\geq 1$ year as of November 1, 2015) in 300 areas, were stratified and randomly extracted from the general census areas in the Comprehensive Survey of Living Conditions in 2015. The 2015 NHNS consisted of physical examination, dietary survey, and lifestyle questionnaire. A total of 3507 out of 5327 eligible households (65.8\%) and 8583 people participated in the survey. This current study included 5048 adults aged 18-64 years. We excluded participants with missing data required for analysis in the present study, such as dietary information $(n=1127)$, body weight $(n=592)$, smoking status or/and habitual alcohol consumption $(n=270)$. Moreover, we excluded those with missing data on the frequency of eating out and take-away meals $(n=5)$. We also excluded those who skipped breakfast, lunch, or/and dinner $(n=749)$, because meal skipping may affect nutrient and food intakes $[31,32]$, and lactating or pregnant women who may have changed their usual dietary habits $(n=84)$ [33]. Thus, the final participants consisted of 2221 Japanese adults aged 18-64 years (921 men and 1300 women).

The permission to use the 2015 NHNS data was obtained from the Ministry of Health, Labour, and Welfare, and only anonymised information was availed for this study. As this survey was conducted according to the Health Promotion Act, all participants gave informed consent to the local government, and approval from Institutional Review Board was not required.

\section{Dietary assessment}

Dietary intake data was collected using a one-day semiweighed household dietary record administered in November 2015, excluding Sundays and public holidays. 
Prior to completing the survey, trained fieldworkers (mainly registered dieticians) provided an outline of the survey and explained to the participants how to complete the dietary record. The main record-keepers in the household (members who are usually responsible for preparing meals) were instructed to weigh all foods and beverages consumed by the household members and the amount of food waste and leftovers and record their names and weights on recording forms. Additionally, the main record-keepers recorded the approximate proportions of the food consumed by each household member when members shared foods from the same dish to enable estimation of individual intake. If weighing was not possible because the meal was consumed away from the home, the portion size consumed, or quantity of foods and details of any leftovers was estimated. Also, participants reported the type of meals consumed at breakfast, lunch, and dinner on the recording day according to the following categories: prepared at home, take-away meals (dishes prepared outside home, but eaten at home), eating out at a restaurant and a fast-food store, or other meals prepared outside the home (food served at nursery school, kindergarten, elementary school, junior high school, high school, or workplace). This selection was identified by the main dishes (staple food in case there were no main side dishes).

Trained fieldworkers visited each household and checked for any missing information and errors. In accordance with the survey manual of the NHNS, the trained fieldworkers converted these estimates of portion sizes or quantity of foods into weights of foods and coded each food item, according to the NHNS food number lists based on the Standard Tables of Food Composition in Japan [34] to calculate the intake of energy and nutrients. The trained fieldworkers inputted collected dietary intake data using software specifically developed for the NHNS.

Energy and nutrients were calculated based on the 2010 Standard Tables of Food Composition in Japan, and food items were classified into 17 groups based on the definition of the Standard Tables of Food Composition [34]. In this study, we adjusted the observed dietary intake for energy requirement to minimize errors associated with selfreporting assessment, using the density method. To render the comparison between the reported nutrient intake and the Dietary Reference Intake for Japanese (DRIs) values [35] practically possible, the following calculation was used: energy-adjusted intake (units/day) = observed intake (units/day) $\times$ estimated energy requirement (EER) (kcal/day)/observed energy intake ( $\mathrm{kcal} /$ day). EER for each participant was assumed as when their physical activity level was at the second level in the Japanese DRIs (PAL = 1.75). For protein, total fat, saturated fat, and carbohydrate, percentage of daily energy intake using reported values (crude) for each macronutrient was also calculated. Additionally, food intake values were energy-adjusted using the density method (i.e. their amounts per EER for food groups: energy-adjusted intake (g/day) = observed intake (g/day) $\times$ EER $(\mathrm{kcal} /$ day $) /$ observed energy intake (kcal/day)).

\section{Frequency of consuming meals prepared away from home}

The frequency of consuming meals prepared away from home was assessed by the combination of two questions in the lifestyle questionnaire asking about the frequency of eating out and take-away meals. Participants reported the frequency of eating out and take-away meals (twice a day or more, once a day, 4-6 times per week, 2-3 times per week, once a week, less than once a week, seldom). Figure 1 shows the classification of participants into three groups according to the frequency of consuming meals prepared away from home, based on the previous reports $[3,15,18]$. Participants who answered, "twice a day or more" to either question and those who answered, "once a day," "4-6 times a week" or "2-3 times a week" to both questions were classified into the High group (high frequency of consuming meals prepared away from home). Participants who responded to both questions "once a week," "less than once a week," "seldom" were classified in the Low group (low frequency of consuming meals prepared away from home). If none of

\begin{tabular}{|c|c|c|c|c|}
\hline & & \multicolumn{3}{|c|}{ Take-away meal consumption } \\
\hline & & $\begin{array}{l}\text { Twice a day or } \\
\text { more }\end{array}$ & $\begin{array}{l}\text { Once a day, } 4-6 \\
\text { times a week or 2-3 } \\
\text { times a week }\end{array}$ & $\begin{array}{l}\text { Once a week, less } \\
\text { than once a week, } \\
\text { or seldom }\end{array}$ \\
\hline \multirow{3}{*}{ 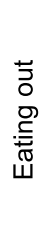 } & Twice a day or more & \multicolumn{3}{|c|}{$\begin{array}{l}\text { Frequency of consuming meals prepared away from home : } \\
\text { High }\end{array}$} \\
\hline & $\begin{array}{l}\text { Once a day, 4-6 times a } \\
\text { week or 2-3 times a week }\end{array}$ & & & Middle \\
\hline & $\begin{array}{l}\text { Once a week, less than } \\
\text { once a week, or seldom }\end{array}$ & & Middle & Low \\
\hline
\end{tabular}

Fig. 1 Classification of study participants based on frequency of consuming meals prepared away from home 
the above applies to those, participants were classified into the Moderate group.

\section{Determination of inadequate nutrient intake}

Inadequate intake of each nutrient was determined by comparing energy-adjusted nutrient levels with the relevant dietary reference value according to the Japanese DRIs, using a previously reported method [36-38]. In the Japanese DRIs, different types of dietary reference values were established according to their purpose. The estimated average requirement (EAR) is set to prevent insufficient intake of nutrients, whereas the tentative dietary goal (DG) to prevent lifestyle-related diseases is set to prevent non-communicable diseases.

Nutrient intake inadequacy was defined as follows: energy-adjusted intake level below EAR was considered as inadequate using the cut-point method for the following 14 nutrients with known EARs: protein, vitamin A (as retinol activity equivalents), vitamin $B_{1}$, vitamin $B_{2}$, niacin (as niacin equivalent), vitamin $B_{6}$, vitamin $B_{12}$, folate, vitamin $C$, calcium, magnesium, iron, zinc, and copper. Regarding iron intake in menstruating women, we applied the value $<9.3 \mathrm{mg} /$ day as recommended by the World Health Organization (WHO) (bioavailability of iron as $15 \%$, probability of inadequacy as $50 \%$ ) [39] for women aged 20-49 years because the cut-point method is less applicable to these populations [40, 41]. For the following seven nutrients, the intake level (energy-adjusted intake level for total dietary fiber, sodium (as saltequivalent) and potassium) outside the range of DG values was considered as inadequate: protein (as \% energy: $13-20 \%$ ), total fat (as \% energy: $20-30 \%$ ), saturated fat (as \% energy: $7 \%$ or less), carbohydrate (as \% energy: $50-65 \%$ ), total dietary fiber (man; $20 \mathrm{~g} /$ day or more, woman; $18 \mathrm{~g} /$ day or more), sodium (as salt-equivalent: man; less than $8.0 \mathrm{~g} /$ day, woman; less than $7.0 \mathrm{~g} /$ day), and potassium (man; $3000 \mathrm{mg} /$ day or more, woman; $2600 \mathrm{mg} /$ day or more).

\section{Other variables}

Body height (to the nearest $0.1 \mathrm{~cm}$ ) and weight (to the nearest $0.1 \mathrm{~kg}$ ) were measured for approximately $90 \%$ of the participants by trained field workers according to standardised procedures. For the remaining participants, height and weight were measured either by other household members at home or were self-reported. BMI was calculated as weight $(\mathrm{kg})$ divided by height $(\mathrm{m})$ squared. Smoking status and alcohol drinking habits during the preceding month were assessed by a self-administered questionnaire.

\section{Statistical analysis}

All statistical analyses were stratified by sex. The differences in characteristics among three groups according to the frequency of consuming meals prepared away from home were compared using the chi-square test for categorical variables and analysis of variance (ANOVA) for continuous variables. Differences in daily energyadjusted nutrients and food group intake among the three groups according to the frequency of consuming meals prepared away from home were assessed by ANOVA in the crude model and a covariate analysis (ANCOVA) in the adjusted model. Dunnett test, with the Low group as reference, was performed in the posthoc test. The nutritional inadequacy of each nutrient intake was represented as the proportion of participants whose energy-adjusted intake was below the EAR or outside the range of the DG in each group. Logistic regression analysis was used to examine the difference in the prevalence of meeting DRIs based on the High and Moderate groups according to the frequency of consuming meals prepared away from home compared with the Low group in the crude and adjusted model. Confounding factors considered in the adjusted model were age category (18-34, 35-50, and 51-64 years), occupation (professional/manager, sales/service/clerical, security/ transportation/labour, student, housekeeper, and not in paid employment), living alone or not (yes or no), region (Hokkaido/Tohoku, Kanto, Hokuriku/Tokai, Kinki, Shikoku/Chugoku, Kyusyu), current smoker (yes or no) and habitual alcohol drinker (yes or no), which was reported as a factor affecting the frequency of consuming meals prepared away from home $[8,42]$. All statistical analyses were performed with SAS statistical software, version 9.4 (SAS Institute Inc., Cary, NC, USA). All reported $P$ values were two-tailed, with a $P$-value $<0.05$ considered statistically significant.

\section{Results}

Table 1 shows the basic characteristics of participants according to their frequency of consuming meals prepared away from home. The proportion of participants classified into the High, Moderate, and Low groups were $14.7,50.5$, and $34.9 \%$ for men, and $6.3,46.9$, and $46.8 \%$ for women, respectively. The mean age in the Low group was significantly higher than other groups $(p=0.001$ in men and $p=0.004$ in women). Additionally, there were more men and women living alone in the High group $(p<0.001)$. The residing region significantly differed in women, with more women in the High group living in the urban Kanto area $(p=0.002)$. In the High group, approximately $90 \%$ of men and $70 \%$ of women reported eating out or having take-away meals in their one-day dietary records as well, which showed a significantly higher proportion compared to other groups $(p<0.001)$. There were no differences in mean BMI, type of occupation, current smoking and consumption of snacks across the groups in both men and women. 
Table 1 Characteristics of 2221 Japanese adults aged 18-64 years according to their frequency of consuming meals prepared away from home [Mean (SD) or n (\%)]

\begin{tabular}{|c|c|c|c|c|c|c|c|c|c|c|c|c|c|c|}
\hline \multirow{4}{*}{$\frac{}{\text { Age (years), Mean (SD) }}$} & \multicolumn{7}{|l|}{ Men } & \multicolumn{7}{|c|}{ Women } \\
\hline & \multicolumn{6}{|c|}{$\begin{array}{l}\text { Frequency of consuming meals } \\
\text { prepared away from home }\end{array}$} & \multirow{3}{*}{$\begin{array}{r}\mathrm{p}^{*} \\
0.001\end{array}$} & \multicolumn{6}{|c|}{$\begin{array}{l}\text { Frequency of consuming meals } \\
\text { prepared away from home }\end{array}$} & \multirow[t]{2}{*}{$\mathrm{p}^{*}$} \\
\hline & \multicolumn{2}{|c|}{$\begin{array}{l}\text { Low } \\
(n=321)\end{array}$} & \multicolumn{2}{|c|}{$\begin{array}{l}\text { Moderate } \\
(n=465)\end{array}$} & \multicolumn{2}{|c|}{$\begin{array}{l}\text { High } \\
(n=135)\end{array}$} & & \multicolumn{2}{|c|}{$\begin{array}{l}\text { Low } \\
(n=608)\end{array}$} & \multicolumn{2}{|c|}{$\begin{array}{l}\text { Moderate } \\
(n=610)\end{array}$} & \multicolumn{2}{|c|}{$\begin{array}{l}\text { High } \\
(n=82)\end{array}$} & \\
\hline & 49.6 & $(11.8)$ & 47.5 & $(12.4)$ & 44.8 & $(12.1)$ & & 48.8 & $(11.0)$ & 47.5 & $(11.7)$ & 44.7 & $(11.4)$ & 0.004 \\
\hline Age category, n (\%) & & & & & & & 0.005 & & & & & & & 0.016 \\
\hline 18-34 years & 44 & $(13.7)$ & 82 & $(17.6)$ & 33 & $(24.4)$ & & 67 & $(11.0)$ & 95 & (15.6) & 16 & $(19.5)$ & \\
\hline $35-50$ years & 103 & $(32.1)$ & 173 & $(37.2)$ & 52 & $(38.5)$ & & 244 & $(40.1)$ & 241 & (39.5) & 39 & $(47.6)$ & \\
\hline $51-64$ years & 174 & $(54.2)$ & 210 & $(45.2)$ & 50 & $(37.0)$ & & 297 & $(48.9)$ & 274 & $(44.9)$ & 27 & (32.9) & \\
\hline Body mass index $\left(\mathrm{kg} / \mathrm{m}^{2}\right)$, Mean (SD) & 23.8 & (3.4) & 23.8 & (3.5) & 24.5 & $(4.4)$ & 0.132 & 22.0 & (3.4) & 22.1 & (3.7) & 22.0 & (3.8) & 0.909 \\
\hline Body mass index category, n (\%) & & & & & & & 0.269 & & & & & & & 0.190 \\
\hline Underweight $(<18.5)$ & 6 & $(1.9)$ & 19 & $(4.1)$ & 5 & (3.7) & & 59 & $(9.7)$ & 80 & $(13.1)$ & 13 & (15.9) & \\
\hline Normal (18.5-25) & 202 & (62.9) & 302 & $(65.0)$ & 80 & (59.3) & & 449 & (73.9) & 424 & $(69.5)$ & 53 & $(64.6)$ & \\
\hline Overweight ( $25 \leq)$ & 113 & $(35.2)$ & 144 & $(31.0)$ & 50 & $(37.0)$ & & 100 & $(16.5)$ & 106 & $(17.4)$ & 16 & (19.5) & \\
\hline Occupation, n (\%) & & & & & & & 0.104 & & & & & & & 0.097 \\
\hline Professional / manager & 105 & (32.7) & 162 & $(34.8)$ & 55 & $(40.7)$ & & 99 & (16.3) & 84 & $(13.8)$ & 18 & $(22.0)$ & \\
\hline Sales / service / clerical & 65 & (20.3) & 117 & $(25.2)$ & 36 & $(26.7)$ & & 232 & $(38.2)$ & 263 & $(43.1)$ & 38 & $(46.3)$ & \\
\hline Security / transportation / labour & 119 & $(37.1)$ & 135 & $(29.0)$ & 29 & (21.5) & & 66 & $(10.9)$ & 45 & $(7.4)$ & 6 & $(7.3)$ & \\
\hline Student & 3 & (0.9) & 10 & $(2.2)$ & 4 & (3.0) & & 7 & $(1.2)$ & 14 & $(2.3)$ & 2 & (2.4) & \\
\hline Housekeeper & 3 & $(0.9)$ & 6 & (1.3) & 2 & $(1.5)$ & & 193 & (31.7) & 194 & $(31.8)$ & 17 & (20.7) & \\
\hline Not in paid employment & 26 & $(8.1)$ & 35 & $(7.5)$ & 9 & $(6.7)$ & & 11 & $(1.8)$ & 10 & (1.6) & 1 & $(1.2)$ & \\
\hline Living alone, n (\%) & 11 & (3.4) & 44 & (9.5) & 22 & (16.3) & $<0.001$ & 39 & $(6.4)$ & 41 & $(6.7)$ & 14 & $(17.1)$ & 0.002 \\
\hline Region, n (\%) & & & & & & & 0.093 & & & & & & & 0.002 \\
\hline Hokkaido and Tohoku & 41 & $(12.8)$ & 47 & $(10.1)$ & 15 & (11.1) & & 63 & $(10.4)$ & 56 & $(9.2)$ & 13 & (15.9) & \\
\hline Kanto & 89 & $(27.7)$ & 176 & (37.9) & 42 & $(31.1)$ & & 172 & (28.3) & 207 & (33.9) & 36 & $(43.9)$ & \\
\hline Hokuriku and Tokai & 60 & $(18.7)$ & 81 & $(17.4)$ & 21 & (15.6) & & 101 & (16.6) & 132 & $(21.6)$ & 15 & (18.3) & \\
\hline Kinki & 53 & $(16.5)$ & 82 & $(17.6)$ & 31 & (23.0) & & 126 & (20.7) & 100 & (16.4) & 10 & $(12.2)$ & \\
\hline Shikoku and Chugoku & 39 & $(12.2)$ & 34 & (7.3) & 11 & $(8.2)$ & & 67 & $(11.0)$ & 54 & (8.9) & 3 & (3.7) & \\
\hline Kyusyu & 39 & $(12.2)$ & 45 & (9.7) & 15 & $(11.1)$ & & 79 & (13.0) & 61 & $(10.0)$ & 5 & $(6.1)$ & \\
\hline Current smoker, n (\%) & 102 & $(31.8)$ & 140 & $(30.1)$ & 54 & $(40.0)$ & 0.094 & 47 & $(7.7)$ & 55 & $(9.0)$ & 7 & $(8.5)$ & 0.720 \\
\hline Habitual alcohol drinker, n (\%) & 117 & $(36.5)$ & 172 & $(37.0)$ & 45 & (33.3) & 0.736 & 49 & $(8.1)$ & 77 & (12.6) & 10 & $(12.2)$ & 0.029 \\
\hline $\begin{array}{l}\text { Frequency of consuming meals prepared away from } \\
\text { home on the dietary recording day }\end{array}$ & & & & & & & $<0.001$ & & & & & & & $<0.001$ \\
\hline 2 times or more, $\mathrm{n}(\%)$ & 18 & $(5.6)$ & 67 & $(14.4)$ & 52 & $(38.5)$ & & 14 & $(2.3)$ & 49 & $(8.0)$ & 15 & (18.3) & \\
\hline Once, n (\%) & 106 & $(33.0)$ & 219 & $(47.1)$ & 68 & $(50.4)$ & & 128 & (21.1) & 233 & $(38.2)$ & 41 & $(50.0)$ & \\
\hline None, n (\%) & 197 & $(61.4)$ & 179 & $(38.5)$ & 15 & $(11.1)$ & & 466 & (76.6) & 328 & $(53.8)$ & 26 & $(31.7)$ & \\
\hline $\begin{array}{l}\text { Consumption of snacks on the dietary recording } \\
\text { day, } n(\%)\end{array}$ & 203 & $(63.2)$ & 293 & (63.0) & 82 & (60.7) & 0.870 & 476 & (78.3) & 460 & (75.4) & 55 & (67.1) & 0.066 \\
\hline
\end{tabular}

SD standard deviation

* Means for continuous values were compared by an analysis of variance and proportions for categorical values were compared by the chi-square test between High, Moderate and Low groups

Table 2 shows the energy-adjusted nutrient intakes on the dietary recording day according to the frequency of consuming meals prepared away from home. Among men, the energy-adjusted intake of protein, calcium, iron, copper, dietary fiber and potassium was significantly lower in the High group than in the Low group ( $p=0.020,0.044,0.008,0.027,0.002$ and 0.004, respectively). In women, the energy-adjusted intake of folate, calcium, magnesium, iron and potassium in the Moderate group was significantly lower than in the Low 
Table 2 Energy-adjusted nutrient intakes on the dietary recording day among 2221 Japanese adults aged 18-64 years according to frequency of consuming meals prepared away from home [Mean (SD)]†

\begin{tabular}{|c|c|c|c|c|c|c|c|c|c|c|c|c|c|c|c|c|}
\hline \multirow[b]{4}{*}{ Energy, kcal/d } & \multicolumn{8}{|l|}{ Men } & \multicolumn{8}{|c|}{ Women } \\
\hline & \multicolumn{6}{|c|}{$\begin{array}{l}\text { Frequency of consuming meals } \\
\text { prepared away from home }\end{array}$} & \multirow{3}{*}{$\begin{array}{l}P^{\|} \\
0.454\end{array}$} & \multirow{3}{*}{$\begin{array}{l}P^{\pi} \\
0.282\end{array}$} & \multicolumn{6}{|c|}{$\begin{array}{l}\text { Frequency of consuming meals } \\
\text { prepared away from home }\end{array}$} & \multirow{3}{*}{$\begin{array}{l}P^{\|} \\
0.771\end{array}$} & \multirow{3}{*}{$\begin{array}{l}P^{\text {I }} \\
0.710\end{array}$} \\
\hline & \multicolumn{2}{|c|}{$\begin{array}{l}\text { Low } \\
(n=321)\end{array}$} & \multicolumn{2}{|c|}{$\begin{array}{l}\text { Moderate } \\
(n=465)\end{array}$} & \multicolumn{2}{|c|}{$\begin{array}{l}\text { High } \\
(n=135)\end{array}$} & & & \multicolumn{2}{|c|}{$\begin{array}{l}\text { Low } \\
(n=608)\end{array}$} & \multicolumn{2}{|c|}{$\begin{array}{l}\text { Moderate } \\
(n=610)\end{array}$} & \multicolumn{2}{|c|}{$\begin{array}{l}\text { High } \\
(n=82)\end{array}$} & & \\
\hline & 2314 & $(592)$ & 2302 & $(571)$ & 2241 & (554) & & & 1771 & $(430)$ & 1785 & (423) & 1799 & (499) & & \\
\hline \multicolumn{17}{|l|}{ Nutrients with EAR } \\
\hline Protein, g/d & 91 & (18) & 91 & (17) & 87 & $(19)^{*}$ & 0.031 & 0.020 & 73 & (14) & 73 & (13) & 72 & (15) & 0.598 & 0.782 \\
\hline \multicolumn{17}{|l|}{ Vitamins } \\
\hline Vitamin A, $\mu \mathrm{g}$ RE/d & 614 & (727) & 662 & (751) & 527 & (366) & 0.136 & 0.181 & 617 & (910) & 559 & $(424)$ & 469 & (248) & 0.111 & 0.084 \\
\hline Vitamin $B_{1}, \mathrm{mg} / \mathrm{d}$ & 1.1 & $(0.41)$ & 1.1 & $(0.42)$ & 1.1 & $(0.44)$ & 0.455 & 0.319 & 0.9 & $(0.31)$ & 0.90 & $(0.30)$ & 0.93 & $(0.34)$ & 0.653 & 0.646 \\
\hline Vitamin $B_{2}, \mathrm{mg} / \mathrm{d}$ & 1.4 & $(0.46)$ & 1.4 & $(0.48)$ & 1.4 & $(0.51)$ & 0.524 & 0.453 & 1.3 & $(0.46)$ & 1.2 & $(0.39)$ & 1.3 & $(0.48)$ & 0.290 & 0.388 \\
\hline Niacin, mg/d & 35 & (9.8) & 36 & $(11.2)$ & 33 & $(10.5)$ & 0.058 & 0.075 & 28 & (7.6) & 28 & $(7.5)$ & 28 & (8.5) & 0.987 & 0.964 \\
\hline Vitamin $B_{6}, \mathrm{mg} / \mathrm{d}$ & 1.4 & $(0.42)$ & 1.4 & $(0.42)$ & 1.3 & $(0.69)$ & 0.135 & 0.291 & 1.2 & $(0.38)$ & 1.2 & $(0.37)$ & 1.1 & $(0.37)$ & 0.569 & 0.724 \\
\hline Vitamin $B_{12}, \mu \mathrm{g} / \mathrm{d}$ & 7.7 & $(7.7)$ & 7.8 & (7.7) & 6.5 & $(6.2)$ & 0.184 & 0.333 & 6.0 & $(6.1)$ & 5.8 & $(6.1)$ & 6.2 & (5.5) & 0.756 & 0.612 \\
\hline Folate, $\mu \mathrm{g} / \mathrm{d}$ & 352 & (135) & 363 & (153) & 334 & (148) & 0.120 & 0.225 & 335 & (144) & 319 & $(123)^{*}$ & 307 & (119) & 0.044 & 0.04 \\
\hline Vitamin C, mg/d & 103 & (70) & 103 & (66) & 87 & (61) & 0.032 & 0.162 & 110 & (71) & 103 & (69) & 92 & (71) & 0.040 & 0.128 \\
\hline \multicolumn{17}{|l|}{ Minerals } \\
\hline Calcium, mg/d & 580 & (258) & 578 & (263) & 519 & $(245)^{*}$ & 0.045 & 0.044 & 561 & (230) & 525 & $(223)^{*}$ & 523 & (243) & 0.019 & 0.036 \\
\hline Magnesium, mg/d & 315 & (93) & 315 & (88) & 294 & (89) & 0.046 & 0.084 & 271 & (75) & 260 & $(71)^{*}$ & 256 & (74) & 0.011 & 0.01 \\
\hline Iron, mg/d & 9.5 & (2.9) & 9.7 & $(2.8)$ & 8.9 & $(2.6)^{*}$ & 0.007 & 0.008 & 8.6 & $(2.8)$ & 8.0 & $(2.2)^{*}$ & 7.9 & (2.5) & $<0.001$ & 0.00 \\
\hline Zinc, mg/d & 10.7 & $(2.2)$ & 10.8 & $(2.5)$ & 10.5 & $(2.5)$ & 0.635 & 0.398 & 8.4 & $(1.8)$ & 8.4 & $(1.8)$ & 8.2 & (1.9) & 0.600 & 0.684 \\
\hline Copper, mg/d & 1.5 & $(0.32)$ & 1.5 & $(0.34)$ & 1.4 & $(0.38)^{*}$ & 0.029 & 0.027 & 1.2 & $(0.29)$ & 1.2 & $(0.29)$ & 1.2 & $(0.34)$ & 0.061 & 0.060 \\
\hline \multicolumn{17}{|l|}{ Nutrients with DG } \\
\hline Protein, \%energy & 14.3 & (2.9) & 14.3 & (2.6) & 13.5 & $(3.0)^{*}$ & 0.005 & 0.015 & 15.0 & (3.0) & 14.9 & (2.8) & 14.6 & (3.0) & 0.451 & 0.800 \\
\hline Fat, \%energy & 25.1 & $(6.7)$ & 26.3 & (6.9) & 26.9 & (6.8) & 0.020 & 0.141 & 28.0 & $(7.1)$ & 28.8 & $(7.2)$ & 28.5 & (7.3) & 0.154 & 0.166 \\
\hline Saturated fat, \%energy & 6.6 & $(2.4)$ & 6.8 & $(2.4)$ & 7.0 & $(2.7)$ & 0.212 & 0.673 & 7.6 & (2.6) & 7.9 & $(2.8)$ & 7.8 & (2.8) & 0.165 & 0.129 \\
\hline Carbohydrate, \%energy & 60.5 & (8.0) & 59.5 & $(8.1)$ & 59.7 & $(7.2)$ & 0.173 & 0.283 & 57.0 & $(8.1)$ & 56.3 & $(8.1)$ & 56.8 & $(8.4)$ & 0.349 & 0.322 \\
\hline Dietary fiber, g/day & 18.1 & (6.8) & 17.9 & (6.6) & 15.9 & $(5.5)^{*}$ & 0.002 & 0.002 & 16.5 & $(6.0)$ & 15.6 & $(6.0)^{*}$ & 14.8 & $(5.1)^{*}$ & 0.004 & 0.005 \\
\hline Sodium (salt-equivalent), day & 13.0 & $(4.1)$ & 13.1 & $(4.2)$ & 13.4 & (3.9) & 0.603 & 0.760 & 10.3 & (3.4) & 10.3 & (3.1) & 10.6 & (3.9) & 0.811 & 0.906 \\
\hline Potassium, mg/d & 2831 & $(832)$ & 2799 & $(801)$ & 2547 & $(908)^{*}$ & 0.003 & 0.004 & 2545 & (739) & 2444 & $(704)^{*}$ & 2372 & $(705)$ & 0.016 & 0.02 \\
\hline
\end{tabular}

$D G$ tentative dietary goal for preventing lifestyle-related disease, EAR estimated average requirement, $S D$ standard deviation

${ }^{+}$Nutrients expressed as amount per day were energy-adjusted by using the following equation: energy-adjusted intake (units/day)=observed dietary intake (units/day) $\times$ estimated energy requirement $(\mathrm{kcal} /$ day)/observed energy intake (kcal/day)

₹ Sum of retinol, $\beta$-carotene/12, a-carotene/24, and cryptoxanthin/24

$\S$ Sum of niacin and protein $/ 6000$

"The $p$ values are shown for an analysis of variance to analyze differences of nutrient intake between three groups

"The $p$ values are shown for covariate analysis to analyze difference of nutrient intake between three groups adjusted for confounding variables of age category (18-34, 35-50, and 51-64 years), occupation (professional / manager, sales / service / clerical, security / transportation / labour, student, housekeeper, and not in paid employment), living alone (yes or no), region (Hokkaido and Tohoku, Kanto, Hokuriku and Tokai, Kinki, Shikoku and Chugoku and Kyusyu), current smoker (yes or no) and habitual alcohol drinker (yes or no). ${ }^{*}$ There is significant difference by Dunnett test compared with Low group in the adjusted model

group ( $p=0.046,0.036,0.014,0.001$ and 0.026 , respectively). Energy-adjusted dietary fiber intake was higher in the Low group compared the Moderate and High groups $(p=0.005)$.

The multivariate-adjusted odds ratios (ORs) for nutrient intake inadequacy according to the frequency of consuming meals prepared away from home are shown in Table 3. Most men and women had adequate intake of protein, niacin and copper in comparison to EAR.
Among men, the proportion of those having inadequate intake of iron, protein \%energy, dietary fiber, and potassium in the High group was significantly higher than in the Low group. The multivariate-adjusted ORs [95\% confidence interval $(\mathrm{CI})$ ] for inadequacy of iron, protein \%energy, dietary fiber, and potassium in the High group compared with the Low group (reference) were 2.03 [1.03-4.01], 1.58 [1.03-2.40], 1.91 [1.17-3.12], and 2.17 [1.33-3.55], respectively. Women in the High group 
Table 3 Multivariate-adjusted ORs for the presence of inadequate nutrient intake (based on EAR and DG) compared with the meeting EAR and DG for frequency of consuming meals prepared away from home among 2221 Japanese adults aged 18-64 years

\begin{tabular}{|c|c|c|c|c|c|c|c|c|c|c|c|c|}
\hline \multirow{3}{*}{$\begin{array}{l}\text { Nutrients with EAR } \\
\end{array}$} & \multicolumn{6}{|c|}{ Men } & \multirow{2}{*}{\multicolumn{6}{|c|}{$\begin{array}{l}\text { Women } \\
\text { Frequency of consuming meals } \\
\text { prepared away from home }\end{array}$}} \\
\hline & \multicolumn{6}{|c|}{$\begin{array}{l}\text { Frequency of consuming meals } \\
\text { prepared away from home }\end{array}$} & & & & & & \\
\hline & \multicolumn{2}{|c|}{$\begin{array}{l}\text { Low } \\
(n=321)\end{array}$} & \multicolumn{2}{|c|}{$\begin{array}{l}\text { Moderate } \\
(n=465)\end{array}$} & \multicolumn{2}{|c|}{$\begin{array}{l}\text { High } \\
(n=135)\end{array}$} & \multicolumn{2}{|c|}{$\begin{array}{l}\text { Low } \\
(n=608)\end{array}$} & \multicolumn{2}{|c|}{$\begin{array}{l}\text { Moderate } \\
(n=610)\end{array}$} & \multicolumn{2}{|c|}{$\begin{array}{l}\text { High } \\
(n=82)\end{array}$} \\
\hline Protein, $n, \%$ & 3 & 0.9 & 2 & \multirow[t]{3}{*}{0.4} & 0 & \multirow[t]{3}{*}{0} & 3 & \multirow[t]{3}{*}{0.2} & 0 & \multirow[t]{3}{*}{0} & 0 & \multirow[t]{3}{*}{0} \\
\hline Crude OR (95\% Cl) & \multicolumn{2}{|l|}{-} & \multirow[t]{2}{*}{-} & & \multirow[t]{2}{*}{ - } & & \multirow[t]{2}{*}{-} & & \multirow[t]{2}{*}{-} & & \multirow[t]{2}{*}{-} & \\
\hline Adjusted OR (95\% Cl) & - & & & & & & & & & & & \\
\hline Vitamins & & & & & & & & & & & & \\
\hline Vitamin A, n, \% & 216 & 67.3 & 289 & 62.2 & 100 & 74.1 & 325 & 53.5 & 325 & 53.3 & 48 & 58.5 \\
\hline Crude OR (95\% Cl) & 1.00 & ference) & 0.80 & 9-1.08) & 1.39 & 9-2.18) & 1.00 & ference) & 0.99 & $-1.24)$ & 1.23 & $7-1.96)$ \\
\hline Adjusted OR (95\% Cl) & 1.00 & ference) & 0.81 & $0-1.10)$ & 1.41 & 8-2.24) & 1.00 & ference) & 0.97 & $-1.22)$ & 1.18 & 3-1.90) \\
\hline Vitamin $B_{1}, n, \%$ & 197 & 61.4 & 288 & 61.9 & 96 & 71.1 & 362 & 59.5 & 362 & 59.3 & 43 & 52.4 \\
\hline Crude OR (95\% Cl) & 1.00 & erence) & 1.02 & $6-1.37)$ & 1.55 & $0-2.39)$ & 1.00 & ference) & 0.99 & $-1.25)$ & 0.75 & 7-1.19) \\
\hline Adjusted OR (95\% Cl) & 1.00 & Fence) & 1.00 & 4-1.34) & 1.44 & $2-2.25)$ & 1.00 & ference) & 1.01 & $-1.27)$ & 0.77 & 8-1.23) \\
\hline Vitamin $B_{2}, n, \%$ & 136 & 42.4 & 182 & 39.1 & 70 & 51.9 & 178 & 29.3 & 173 & 28.4 & 25 & 30.5 \\
\hline Crude OR (95\% Cl) & 1.00 & erence) & 0.88 & $6-1.17)$ & 1.47 & 8-2.19) & 1.00 & ference) & 0.96 & $-1.23)$ & 1.06 & 4-1.75) \\
\hline Adjusted OR (95\% Cl) & 1.00 & Fence) & 0.86 & 4-1.16) & 1.46 & $6-2.22)$ & 1.00 & ference) & 0.92 & $-1.18)$ & 1.00 & $0-1.66)$ \\
\hline Niacin, $n, \%$ & 1 & 0.3 & 0 & 0 & 0 & 0 & 0 & 0 & 0 & 0 & 0 & 0 \\
\hline Crude OR (95\% Cl) & - & & - & & - & & - & & - & & - & \\
\hline Adjusted OR (95\% Cl) & - & & - & & - & & - & & - & & - & \\
\hline Vitamin $B_{6}, n, \%$ & 107 & 33.3 & 154 & 33.1 & 59 & 43.7 & 212 & 34.9 & 221 & 36.2 & 32 & 39.0 \\
\hline Crude OR (95\% Cl) & 1.00 & Fence) & 0.99 & (3-1.34) & 1.55 & $03-2.34)$ & 1.00 & ference) & 1.06 & (-1.34) & 1.20 & 4-1.92) \\
\hline Adjusted OR (95\% Cl) & 1.00 & erence) & 0.95 & (0-1.30) & 1.36 & 8-2.09) & 1.00 & ference) & 1.04 & $-1.32)$ & 1.17 & 2-1.90) \\
\hline Vitamin $B_{12}, n, \%$ & 46 & 14.3 & 53 & 11.4 & 18 & 13.3 & 138 & 22.7 & 123 & 20.2 & 17 & 20.7 \\
\hline Crude OR (95\% Cl) & 1.00 & erence) & 0.77 & $0-1.17)$ & 0.92 & $1-1.65)$ & 1.00 & ference) & 0.86 & $(-1.13)$ & 0.89 & $1-1.57)$ \\
\hline Adjusted OR (95\% Cl) & 1.00 & erence) & 0.75 & $9-1.15)$ & 0.85 & $6-1.56)$ & 1.00 & ference) & 0.82 & $-1.07)$ & 0.76 & $3-1.36)$ \\
\hline Folate, $n, \%$ & 26 & 8.1 & 46 & 9.9 & 20 & 14.8 & 78 & 12.8 & 85 & 13.9 & 18 & 22.0 \\
\hline Crude OR (95\% Cl) & 1.00 & erence) & 1.25 & $5-2.06)$ & 1.97 & $06-3.67)$ & 1.00 & ference) & 1.10 & $9-1.53)$ & 1.91 & (8-3.39) \\
\hline Adjusted OR (95\% Cl) & 1.00 & Fence) & 1.15 & 9-1.93) & 1.65 & $7-3.16)$ & 1.00 & ference) & 1.03 & -1.45) & 1.80 & $0-3.27)$ \\
\hline Vitamin C, n, \% & 153 & 47.7 & 217 & 46.7 & 79 & 58.5 & 258 & 42.4 & 296 & 48.5 & 48 & 58.5 \\
\hline Crude OR (95\% CI) & 1.00 & Fence) & 0.96 & (2-1.28) & 1.55 & $03-2.33)$ & 1.00 & ference) & 1.28 & $2-1.60)$ & 1.92 & 20-3.06) \\
\hline Adjusted OR (95\% Cl) & 1.00 & Fence) & 0.87 & $5-1.17)$ & 1.25 & 2-1.92) & 1.00 & ference) & 1.20 & $-1.52)$ & 1.72 & $5-2.80)$ \\
\hline Minerals & & & & & & & & & & & & \\
\hline Calcium, $n, \%$ & 189 & 58.9 & 267 & 57.4 & 91 & 67.4 & 327 & 53.8 & 364 & 59.7 & 52 & 63.4 \\
\hline Crude OR (95\% Cl) & 1.00 & erence) & 0.94 & $0-1.26)$ & 1.44 & $5-2.21)$ & 1.00 & ference) & 1.27 & $1-1.60)$ & 1.49 & $3-2.40)$ \\
\hline Adjusted OR $(95 \% \mathrm{Cl})$ & 1.00 & erence) & 0.93 & 9-1.26) & 1.42 & $1-2.20)$ & 1.00 & ference) & 1.24 & $3-1.56)$ & 1.44 & $8-2.35)$ \\
\hline Magnesium, $n, \%$ & 156 & 48.6 & 225 & 48.4 & 76 & 56.3 & 222 & 36.5 & 263 & 43.1 & 34 & 41.5 \\
\hline Crude OR (95\% Cl) & 1.00 & Fence) & 0.99 & $5-1.32)$ & 1.36 & $1-2.04)$ & 1.00 & ference) & 1.32 & $5-1.66)$ & 1.23 & 7-1.97) \\
\hline Adjusted OR (95\% Cl) & 1.00 & ference) & 0.99 & $4-1.33)$ & 1.27 & 3-1.93) & 1.00 & ference) & 1.31 & $3-1.65)$ & 1.22 & $5-1.98)$ \\
\hline Iron, $n, \%$ & 23 & 7.2 & 26 & 5.6 & 19 & 14.1 & 237 & 39.0 & 270 & 44.3 & 44 & 53.7 \\
\hline Crude OR (95\% Cl) & 1.00 & Fence) & 0.77 & $3-1.37)$ & 2.12 & $11-4.04)$ & 1.00 & ference) & 1.24 & $9-1.56)$ & 1.81 & 14-2.88) \\
\hline Adjusted OR $(95 \% \mathrm{Cl})$ & 1.00 & ference) & 0.75 & 1-1.36) & 2.03 & $03-4.01)$ & 1.00 & ference) & 1.13 & $+1.51)$ & 1.35 & $4-2.46)$ \\
\hline Zinc, $n, \%$ & 24 & 7.5 & 42 & 9.0 & 14 & 10.4 & 36 & 5.9 & 26 & 4.3 & 7 & 8.5 \\
\hline
\end{tabular}


Table 3 Multivariate-adjusted ORs for the presence of inadequate nutrient intake (based on EAR and DG) compared with the meeting EAR and DG for frequency of consuming meals prepared away from home among 2221 Japanese adults aged 18-64 years (Continued)

\begin{tabular}{|c|c|c|c|c|c|c|c|c|c|c|c|c|}
\hline & \multicolumn{6}{|l|}{ Men } & \multicolumn{6}{|c|}{ Women } \\
\hline & \multicolumn{6}{|c|}{$\begin{array}{l}\text { Frequency of consuming meals } \\
\text { prepared away from home }\end{array}$} & \multicolumn{6}{|c|}{$\begin{array}{l}\text { Frequency of consuming meals } \\
\text { prepared away from home }\end{array}$} \\
\hline & \multicolumn{2}{|c|}{$\begin{array}{l}\text { Low } \\
(n=321)\end{array}$} & \multicolumn{2}{|c|}{$\begin{array}{l}\text { Moderate } \\
(n=465)\end{array}$} & \multicolumn{2}{|c|}{$\begin{array}{l}\text { High } \\
(n=135)\end{array}$} & \multicolumn{2}{|c|}{$\begin{array}{l}\text { Low } \\
(n=608)\end{array}$} & \multicolumn{2}{|c|}{$\begin{array}{l}\text { Moderate } \\
(n=610)\end{array}$} & \multicolumn{2}{|c|}{$\begin{array}{l}\text { High } \\
(n=82)\end{array}$} \\
\hline Crude OR $(95 \% \mathrm{Cl})$ & \multicolumn{2}{|c|}{1.00 (Reference) } & \multicolumn{2}{|c|}{$1.23(0.73-2.07)$} & \multicolumn{2}{|c|}{$1.43(0.72-2.86)$} & \multicolumn{2}{|c|}{1.00 (Reference) } & \multicolumn{2}{|c|}{$0.71(0.42-1.19)$} & \multicolumn{2}{|c|}{$1.48(0.64-3.45$} \\
\hline Adjusted OR (95\% Cl) & \multicolumn{2}{|c|}{1.00 (Reference) } & \multicolumn{2}{|c|}{$1.24(0.72-2.13)$} & \multicolumn{2}{|c|}{$1.57(0.75-3.29)$} & \multicolumn{2}{|c|}{1.00 (Reference) } & \multicolumn{2}{|c|}{$0.63(0.37-1.08)$} & \multicolumn{2}{|c|}{$1.22(0.50-2.99$} \\
\hline Copper, n, \% & 0 & 0 & 2 & 0.4 & 1 & 0.7 & 2 & 0.3 & 3 & 0.5 & 0 & 0 \\
\hline Crude OR (95\% Cl) & \multicolumn{2}{|l|}{-} & \multicolumn{2}{|l|}{-} & \multicolumn{2}{|l|}{-} & \multicolumn{2}{|l|}{-} & \multicolumn{2}{|l|}{-} & - & \\
\hline Adjusted OR (95\% Cl) & - & & - & & - & & - & & - & & - & \\
\hline Nutrients with DG & & & & & & & & & & & & \\
\hline Protein, $n, \%$ & 110 & 34.3 & 159 & 34.2 & 63 & 46.7 & 182 & 29.9 & 186 & 30.5 & 31 & 37.8 \\
\hline Crude OR $(95 \% \mathrm{Cl})$ & 1.00 & ference) & 1.00 & (4-1.35) & 1.68 & $12-2.53)$ & 1.00 & ference) & 1.03 & $0-1.31)$ & 1.42 & 38-2.30) \\
\hline Adjusted OR (95\% Cl) & 1.00 & ference) & 0.97 & $2-1.32)$ & 1.58 & $03-2.40)$ & 1.00 & ference) & 1.00 & 8-1.28) & 1.30 & $30-2.11)$ \\
\hline Fat, $n, \%$ & 134 & 41.7 & 222 & 47.7 & 61 & 45.2 & 325 & 53.5 & 326 & 53.4 & 49 & 59.8 \\
\hline Crude OR $(95 \% \mathrm{CI})$ & 1.00 & ference) & 1.28 & $6-1.70)$ & 1.15 & 7-1.73) & 1.00 & ference) & 1.00 & $0-1.25)$ & 1.29 & $31-2.07)$ \\
\hline Adjusted OR (95\% Cl) & 1.00 & ference) & 1.28 & $6-1.71)$ & 1.12 & $4-1.71)$ & 1.00 & ference) & 1.00 & $0-1.26)$ & 1.31 & $81-2.11)$ \\
\hline Saturated fat, $n, \%$ & 121 & 37.7 & 200 & 43.0 & 59 & 43.7 & 338 & 55.6 & 361 & 59.2 & 46 & 56.1 \\
\hline Crude OR $(95 \%$ Cl) & 1.00 & ference) & 1.25 & $3-1.67)$ & 1.28 & 5-1.93) & 1.00 & ference) & 1.16 & $2-1.45)$ & 1.02 & 54-1.62) \\
\hline Adjusted OR (95\% Cl) & 1.00 & ference) & 1.23 & $1-1.65)$ & 1.18 & $7-1.80)$ & 1.00 & ference) & 1.15 & $1-1.44)$ & 1.01 & 53-1.61) \\
\hline Carbohydrate, n, \% & 121 & 37.7 & 167 & 35.9 & 43 & 31.9 & 219 & 36.0 & 204 & 33.4 & 32 & 39.0 \\
\hline Crude OR (95\% Cl) & 1.00 & ference) & 0.93 & (9-1.24) & 0.70 & 0-1.18) & 1.00 & ference) & 0.89 & $1-1.13)$ & 1.14 & 71-1.83) \\
\hline Adjusted OR (95\% Cl) & 1.00 & ference) & 0.95 & $0-1.28)$ & 0.80 & $2-1.25)$ & 1.00 & ference) & 0.88 & $9-1.11)$ & 1.22 & 75-1.98) \\
\hline Dietary fiber, $n, \%$ & 211 & 65.7 & 319 & 68.6 & 107 & 79.3 & 399 & 65.6 & 440 & 72.1 & 60 & 73.2 \\
\hline Crude OR (95\% Cl) & 1.00 & ference) & 1.14 & $34-1.54)$ & 1.99 & $24-3.21)$ & 1.00 & ference) & 1.36 & $66-1.73)$ & 1.43 & $85-2.39)$ \\
\hline Adjusted OR (95\% Cl) & 1.00 & ference) & 1.14 & $33-1.56)$ & 1.91 & $17-3.12)$ & 1.00 & ference) & 1.32 & $3-1.69)$ & 1.31 & $77-2.23)$ \\
\hline Sodium (salt-equivalent), $n, \%$ & 297 & 92.5 & 427 & 91.8 & 129 & 95.6 & 522 & 85.9 & 532 & 87.2 & 69 & 84.2 \\
\hline Crude OR (95\% Cl) & 1.00 & ference) & 0.91 & $3-1.55)$ & 1.74 & $9-4.35)$ & 1.00 & ference) & 1.12 & $1-1.56)$ & 0.87 & $46-1.65)$ \\
\hline Adjusted OR (95\% Cl) & 1.00 & ference) & 0.95 & $5-1.64)$ & 1.88 & $3-4.84)$ & 1.00 & ference) & 1.11 & $0-1.55)$ & 0.86 & $45-1.64)$ \\
\hline Potassium, $n, \%$ & 202 & 62.9 & 305 & 65.6 & 107 & 79.3 & 348 & 57.2 & 383 & 62.8 & 49 & 59.8 \\
\hline Crude OR (95\% CI) & 1.00 & ference) & 1.12 & $34-1.51)$ & 2.25 & $40-3.62)$ & 1.00 & ference) & 1.26 & $0-1.59)$ & 0.88 & $55-1.41)$ \\
\hline Adjusted OR (95\% Cl) & 1.00 & ference) & 1.13 & $33-1.53)$ & 2.17 & $33-3.55)$ & 1.00 & ference) & 1.23 & $7-1.56)$ & 1.01 & 52-1.65) \\
\hline
\end{tabular}

$C l$ confidence interval, $D G$ tentative dietary goal for preventing lifestyle-related disease, DRI Dietary Reference Intakes, EAR estimated average requirement, OR odd ratio

Percentage of subjects whose intake was in the range of DG or above the EAR. Each energy-adjusted nutrient intake (units/day) was compared with each DRI value (units/day), using the cut-point method

The probability of inadequacy $>50 \%$ for menstruating women whose bioavailability of iron is $15 \%(<9.3 \mathrm{mg} / \mathrm{d})$ was considered inadequate for women aged 20-49 years

* Adjusted for confounding variables of age category (18-34, 35-50, and 51-64 years), occupation (professional / manager, sales / service / clerical, security / transportation / labour, student, housekeeper, and not in paid employment), living alone (yes or no), region (Hokkaido and Tohoku, Kanto, Hokuriku and Tokai, Kinki, Shikoku and Chugoku and Kyusyu), current smoker (yes or no) and habitual alcohol drinker (yes or no)

were less likely to have adequate vitamin $\mathrm{C}$ intake compared to women in the Low group (OR [95\% CI]; 1.72 [1.05-2.80]). For magnesium and dietary fiber, the multivariate-adjusted ORs were significantly higher in the Moderate group than in the Low group (ORs [95\% CI); 1.31 [1.03-1.65] and 1.32 [1.03-1.69]).
Table 4 shows energy-adjusted food group intakes according to the frequency of consuming meals prepared away from home. For both men and women, energyadjusted vegetable intake in the Low groups was higher than that of the High group $(p=0.004$ and $p=0.012$ in men and women, respectively). Energy-adjusted fat and 
Table 4 Energy-adjusted food group intake on the dietary recording day among 2221 Japanese adults aged 18-64 years according to frequency of consuming meals prepared away from home [Mean (SD)]†

\begin{tabular}{|c|c|c|c|c|c|c|c|c|c|c|}
\hline \multirow{3}{*}{$\begin{array}{l}\text { Food groups (g/ } \\
\text { day) }\end{array}$} & \multicolumn{5}{|l|}{ Men } & \multicolumn{5}{|l|}{ Women } \\
\hline & \multicolumn{3}{|c|}{$\begin{array}{l}\text { Frequency of consuming meals } \\
\text { prepared away from home }\end{array}$} & \multirow[t]{2}{*}{$\mathrm{P}^{\neq}$} & \multirow[t]{2}{*}{$\mathrm{P}^{\S}$} & \multicolumn{3}{|c|}{$\begin{array}{l}\text { Frequency of consuming meals } \\
\text { prepared away from home }\end{array}$} & \multirow[t]{2}{*}{$\mathrm{P}^{\neq}$} & \multirow[t]{2}{*}{$P^{\S}$} \\
\hline & $\begin{array}{l}\text { Low } \\
(n=321)\end{array}$ & $\begin{array}{l}\text { Moderate } \\
(n=465)\end{array}$ & $\begin{array}{l}\text { High } \\
(n=135)\end{array}$ & & & $\begin{array}{l}\text { Low } \\
(n=608)\end{array}$ & $\begin{array}{l}\text { Moderate } \\
(n=610)\end{array}$ & $\begin{array}{l}\text { High } \\
(n=82)\end{array}$ & & \\
\hline Grains & $637.8(178.1)$ & $636.2(181.8)$ & $668.0(196.3)$ & 0.187 & 0.372 & $431.9(143.5)$ & $434.7(143.4)$ & $468.6(171.2)$ & 0.099 & 0.148 \\
\hline Potatoes & 66.5 (79.7) & $56.8(66.6)$ & $52.7(67.7)$ & 0.086 & 0.063 & $59.3(75.8)$ & $44.5(56.0)^{*}$ & $52.6(74.1)$ & 0.001 & 0.002 \\
\hline Sugars & $7.9(11.0)$ & $7.1(8.9)$ & $7.7(8.2)$ & 0.507 & 0.694 & $7.1(8.3)$ & $7.1(8.9)$ & $5.5(6.3)$ & 0.226 & 0.292 \\
\hline Pulses & 79.9 (101.3) & 76.4 (83.3) & $67.9(92.8)$ & 0.441 & 0.526 & 75.4 (91.6) & $64.8(74.7)$ & $65.6(90.3)$ & 0.079 & 0.077 \\
\hline Sesame and nuts & $2.2(5.7)$ & $3.3(12.2)$ & $1.7(5.2)$ & 0.119 & 0.202 & $2.4(5.4)$ & $2.8(8.3)$ & $1.9(4.8)$ & 0.378 & 0.376 \\
\hline Vegetables & $354.3(186.5)$ & $362.4(204.3)$ & $294.2(171.8)^{*}$ & 0.001 & 0.004 & $322.2(171.6)$ & $312.0(161.7)$ & $262.1(160.8)^{*}$ & 0.009 & 0.012 \\
\hline Fruits & $94.6(149.3)$ & $84.0(114.6)$ & $56.4(125.3)$ & 0.016 & 0.076 & 109.8 (120.0) & 104.8 (130.6) & $95.1(123.3)$ & 0.546 & 0.815 \\
\hline Mushrooms & 22.9 (37.9) & $18.6(30.4)$ & $13.1(23.0)^{*}$ & 0.010 & 0.015 & $20.2(28.7)$ & $18.7(30.3)$ & $16.1(25.7)$ & 0.423 & 0.422 \\
\hline Seaweeds & $15.5(25.8)$ & $12.0(19.8)$ & $11.9(20.1)$ & 0.068 & 0.086 & $11.4(21.2)$ & $10.1(19.5)$ & $11.0(22.2)$ & 0.541 & 0.593 \\
\hline Fish and shellfishes & 88.5 (84.6) & $87.7(77.2)$ & 71.7 (78.8) & 0.091 & 0.276 & $67.7(64.0)$ & $66.1(68.2)$ & $67.1(66.9)$ & 0.913 & 0.856 \\
\hline Meat and poultry & $131.1(87.3)$ & $134.8(96.3)$ & $138.2(100.8)$ & 0.739 & 0.988 & $91.5(64.9)$ & $101.1(71.5)^{*}$ & $87.4(66.4)$ & 0.027 & 0.032 \\
\hline Eggs & $48.8(46.7)$ & $45.4(41.3)$ & $43.3(41.2)$ & 0.391 & 0.419 & $41.4(38.0)$ & $39.3(38.0)$ & $38.0(38.5)$ & 0.536 & 0.597 \\
\hline Dairy products & $100.9(143.8)$ & $104.3(139.9)$ & $97.0(163.1)$ & 0.863 & 0.744 & $124.5(140.1)$ & $124.5(130.8)$ & $124.0(135.1)$ & 0.999 & 0.992 \\
\hline Fat and oils & $13.4(10.2)$ & $15.9(11.0)^{*}$ & $17.5(10.8)^{*}$ & $<0.001$ & 0.002 & $11.0(9.0)$ & $12.9(9.5)^{*}$ & $13.2(8.2)$ & 0.001 & 0.001 \\
\hline Confectionaries & $19.5(42.0)$ & $21.6(43.2)$ & $22.6(54.1)$ & 0.728 & 0.806 & 32.9 (47.6) & $28.5(44.3)$ & $31.8(40.5)$ & 0.247 & 0.339 \\
\hline Beverages & $1124.5(684.1)$ & 1146.0 (755.8) & $1207.4(748.5)$ & 0.541 & 0.513 & $939.4(544.3)$ & $930.6(542.6)$ & $1000.7(615.3)$ & 0.554 & 0.491 \\
\hline Seasonings & $115.5(108.0)$ & $135.7(125.0)$ & $147.4(144.0)$ & 0.016 & 0.084 & $84.9(77.4)$ & $93.6(86.4)$ & $97.4(120.0)$ & 0.139 & 0.125 \\
\hline me $p$ values die & & 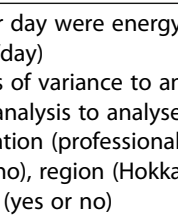 & 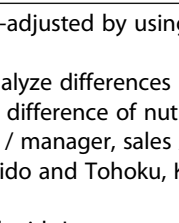 & 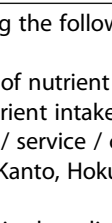 & & $x_{1}$ & 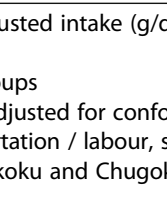 & 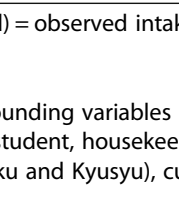 & 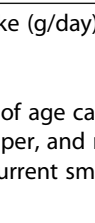 & $\begin{array}{l}\text { egory } \\
\text { ot in } \\
\text { ker }\end{array}$ \\
\hline
\end{tabular}

oil intake in the Low group was lower than the Moderate and High groups among men $(p=0.002)$; significant difference was observed only between the Low and Moderate groups in women. Among men, a higher intake of mushrooms adjusted for energy was observed in the Low group than in the High group $(p=0.015)$. Among women, a higher intake of potatoes and lower intake of meat and poultry adjusted for energy were observed in the Low group than in the Moderate group ( $p=0.002$ and $p=0.032$, respectively).

The association between the frequency of eating out and take-away meals assessed through the one-day dietary record (classified into 3 groups according the frequency of consuming meals prepared away from home; never, once, and twice a day or more) and their nutrient intake inadequacy status is shown in the Supplementary Tables 1-4. Subjects in the "twice or more" group were less likely to have adequate vitamin and mineral intake, except vitamin $B_{1}$, vitamin $B_{12}$, calcium and copper (both men and women), and vitamin $B_{2}$ and vitamin $C$ (only women) compared to the "never" group.

\section{Discussion}

The present study examined the association between the frequency of consuming meals prepared away from home and nutrient intake inadequacy among Japanese adults aged 18-64 years. We found that inadequate intake of dietary fiber, vitamin $\mathrm{C}$ and several minerals was associated with a higher frequency of eating out or takeaway meal. To the best of our knowledge, this study is the first to examine the association between the frequency of consuming meals prepared away from home and nutritional inadequacy, based on dietary reference values among Japanese adults.

In this study, participants were classified into three groups (Low, Moderate, and High group according to the frequency of consuming meals prepared away from home) based on the response to questions about the frequency of eating out and take-away meals. This categorization based on the questionnaire on the frequency of habitual eating out and take-away meals was comparable to that based on the dietary records, as 
shown in Table 1, despite being based on one-day dietary record method. Moreover, the association between the frequency of eating out and take-away meals assessed through the one-day dietary record and nutritional adequacy (Supplementary tables) showed similar results. A higher frequency of consuming meals prepared away from home was associated with less adequate dietary intake. Although the number of vitamins and minerals considered inadequate was larger when the classification using the dietary records was applied compared to that from the lifestyle questionnaire, the two different methods consistently supported our present findings.

Several factors increased the likelihood of eating food prepared away from home. Men and younger people had a higher frequency of consuming meals prepared away from home than others. This is consistent with previous studies that showed a higher frequency of eating out among men and younger adults when compared with that among older adults $[15,43]$, or higher proportion of eating out in men than women [15]. Similarly to a previous study among Japanese university students [37], living alone was associated with a higher frequency of eating meals prepared away from home. In addition, women living in urban areas had more eating out and take-away meals, which is consistent with the result of a previous study in Vietnam [44]. Thus, the current results may indicate that younger adults, especially men, are more likely to consume meals prepared away from home in Japan, as observed in other countries.

The association between the frequency of consuming meals prepared away from home and nutrients intake has been reported in several studies. Studies from Australia and Europe that used 24-h dietary recalls reported that adults with a higher frequency of consumption of foods prepared outside the home had lower intakes of iron and calcium, and vitamin $\mathrm{C}$ and calcium, respectively [2, 13]. Additionally, a review article reported that people with a higher frequency of eating out had lower vitamin $C$, iron and calcium intakes [12]. These reports showed similar results to the current results about energy-adjusted iron and calcium intakes. However, inadequate intake of these nutrients based on dietary reference values was not observed except for iron among men in the present study. According to a previous Japanese study, approximately more than $50 \%$ of Japanese adults had inadequate intake of calcium [45]. Also, another study showed that the proportion of Japanese women who met the standard value of iron intake was low, whereas a large percentage of Japanese men met the standard [46]. Japanese usual insufficient intake status may reflect to the present results, regardless of the frequency of consuming meals prepared away from home. In contrast, Japanese people rarely lack copper and protein [45], which may explain the current results where no difference was observed in the proportion of inadequate intake of these nutrients according to the frequency of consumption meals prepared away from home. Of note, EAR is set for the purpose of avoiding insufficient intake, whereas DG is set for the prevention of non-communicable diseases. It is possible that the definition of inadequacy of each nutrient intake may have determined differently, which suggests a caution of interpretation of the results.

Dietary fiber was the only nutrient that was observed in the inadequacy of intake depending on the frequency of consuming meals prepared away from home both in men and women. This finding is largely consistent with the previous studies that reported the association the frequency of eating out with dietary fiber intake $[2,8]$. In this study, more inadequacy of dietary fiber intake was observed in the High group in men (OR (95\%CI): 1.91 (1.17-3.12)), and the Moderate group in women (OR (95\%CI): 1.32 (1.03-1.69)). It has been reported that women cook more often than men in Japan and other countries $[42,47]$. The dietary fiber intake has been reported to be associated with frequency of cooking and cooking skill [48]. Higher income is associated with a higher frequency of eating out and take-away meals [41]. Additionally, better diets are seen in women compared with men [46], and highly educated individuals have greater dietary fiber and healthy food intake despite more frequent eating out and take-away [8]. These reports may partly explain our present findings that a higher frequency of consuming meals prepared away from home is associated with lower energy-adjusted nutrients intakes and inadequacy of nutrient intake compared with that in the Low group. Also, socioeconomic factor may be one of the important factors associated with consumption of meals prepared away from home. Unfortunately, other than occupation, we could not consider other socioeconomic indicators. While the proportion of occupation (professional, manager, sales, service, and clerics) differed among men and women, and was higher in the High group, there was no significant difference among the groups. Thus, future studies are needed with consideration of socioeconomic factors such as educational background and income level.

The frequency of eating out and take-away meals has been reported to be associated with a lower energyadjusted intake of vegetable and a higher energyadjusted intake of fat and oils $[8,18]$. These results are consistent with the present study. Low intake of vegetables may partly explain the inadequate intake of potassium among men, and inadequate intake of magnesium and vitamin $\mathrm{C}$ among women. Especially, as there has been no report about inadequate intake of potassium 
and magnesium according to the frequency of consuming meals prepared away from home, our results may highlight the need for health promotion interventions for people with a higher frequency of eating out or takeaway meals, as well as for the food industry.

In this study, approximately $45 \%$ of men and $30 \%$ of women regularly ate out or had take-away meal. In Japan, the government has called for voluntary efforts among the food industry to improve the food environment so that people can eat well-balanced meals, whether they eat out or prepare for themselves. Example of such efforts includes "increase in the number of corporations in the food industry that supply food products low in salt and fat." [49] However, the current recommendation hugely focuses on preventing excess intake, and further efforts by the government are needed to increase the population intake of dietary fiber and minerals.

The study had some limitations. First, the participants were randomly selected from nationally representative households in Japan; however, the individual-level response rate was unknown. This might have introduced some bias in the estimation of average intake in Japanese adults. Also, the sample was essentially selected through a cluster of the households; however, it is difficult to treat them with the household cluster in our analysis. Second, a dietary intake derived from one-day weighed dietary record is unlikely to represent the usual intake. Therefore, the variability in the dietary intake of individuals over a period of several days might have influenced the findings. It is noted that the one-day householdbased dietary record method used in NHNS has been compared with individual dietary records among Japanese participants, and the correlation coefficients of the intakes of total energy and macronutrients, such as protein, fat, and carbohydrates were high (0.89 to 0.91). Thus, this method may be valid for the estimation of individual intake [50]. Third, it could have been difficult for participants to accurately weigh food consumption in the case of eating out, take-away, or ready-meal use, unlike when they consumed home-cooked meals and could weigh all the foods and beverages, including the amounts of food waste and leftovers. Therefore, nutrient and food intakes may not have been accurately assessed. Fourth, we limited the participants to those who had three meals a day in the present analysis, because the purpose of this study was to assess nutrient intake and nutrition adequacy by the difference in the frequency of consuming meals prepared away from home. This might have induced some bias in the nutrient intakes. Fifth, we adjusted energy intake using EER assuming physical activity level to be level II for all participants due to the absence of quantitative information about physical activity, based on a previous study [51]. Therefore, it cannot be denied that this may have influenced the results of the current study. Finally, factors other than the frequency of consuming meals prepared away from home may also affect the adequacy of nutrient intake. Future studies should examine the causes of nutrient intake inadequacy.

\section{Conclusions}

This cross-sectional study indicated that Japanese adults aged 18-64 years with a higher frequency of consuming meals prepared away from home were less likely to meet the standard values of dietary fiber, vitamin $C$ and multiple minerals intake. Our findings suggest that these nutrients may be the focus of an interventional approach to improve the nutrient intake status of those with a high frequency of eating out and take away meals among Japanese adults. Further studies targeting food environment, including the food industry, are needed to improve nutritional adequacy for those with a higher frequency of eating out or consuming take-away meals.

\section{Abbreviations}

ANCOVA: Covariate analysis; ANOVA: Analysis of variance; BMl: Body mass index; DG: Tentative dietary goal to prevent lifestyle-related diseases; DRIs: Dietary Reference Intakes for Japanese; EAR: Estimated average requirements; EER: Estimated energy requirement; NHNS: National Health and Nutrition Survey in Japan; OR: Odd ratio; 95\% Cl: 95\% confidence interval

\section{Supplementary Information}

The online version contains supplementary material available at https://doi. org/10.1186/s12937-021-00693-6.

Additional file 1.

\section{Acknowledgments}

Not applicable.

Authors' contributions

All authors designed research. M.M., A.S. and R.T. analyzed the data. M.M. wrote the first draft. A.S., C.O. and E.O. took part in the interpretation of the data and provided critical revisions of the manuscript for important intellectual content. H.T. had primary responsibility for final content. All authors read and approved the final manuscript.

\section{Funding}

This study is part of the Health Japan 21 (the second term) Analysis and Assessment Project, funded by Ministry of Health, Labour, and Welfare. Ministry of Health, Labour, and Welfare had no role in the design, analysis or writing of this article.

\section{Availability of data and materials}

This study was a secondary analysis of the 2015 National Health and Nutrition Survey in Japan and was conducted with the permission of the Ministry of Health, Labour and Welfare, in Japan.

\section{Declarations}

Ethics approval and consent to participate Not applicable.

Consent for publication

Not applicable. 


\section{Competing interests}

The authors declare that they have no competing interests.

Received: 23 May 2020 Accepted: 3 April 2021

Published online: 23 April 2021

\section{References}

1. Beydoun MA, Powell LM, Wang Y. Reduced away-from-home food expenditure and better nutrition knowledge and belief can improve quality of dietary intake among US adults. Public Health Nutr. 2009;12(3):369-81. https://doi.org/10.1017/S1368980008002140.

2. Burns C, Jackson M, Gibbons C, Stoney RM. Foods prepared outside the home: association with selected nutrients and body mass index in adult Australians. Public Health Nutr. 2002;5(3):441-8. https://doi.org/10.1079/ PHN2001316.

3. Goffe L, Rushton S, White M, Adamson A, Adams J. Relationship between mean daily energy intake and frequency of consumption of out-of-home meals in the UK National Diet and nutrition survey. Int J Behav Nutr Phys Act. 2017;14(1):131. https://doi.org/10.1186/s12966-017-0589-5.

4. Ma Y, Bertone ER, Stanek EJ 3rd, Reed GW, Hebert JR, Cohen NL, et al. Association between eating patterns and obesity in a free-living US adult population. Am J Epidemiol. 2003;158(1):85-92. https://doi.org/10.1093/aje/ kwg117.

5. Haines PS, Hungerford DW, Popkin BM, Guilkey DK. Eating patterns and energy and nutrient intakes of US women. J Am Diet Assoc. 1992;92:698704707.

6. Kearney JM, Hulshof KF, Gibney MJ. Eating patterns--temporal distribution, converging and diverging foods, meals eaten inside and outside of the home--implications for developing FBDG. Public Health Nutr. 2001;4(2b): 693-8. https://doi.org/10.1079/PHN2001156.

7. Mattes RD, Donnelly D. Relative contributions of dietary sodium sources. J Am Coll Nutr. 1991;10(4):383-93. https://doi.org/10.1080/07315724.1991.1 0718167.

8. Ljubicic M, Saric MM, Baric IC, Rumbak I, Komes D, Satalic Z, et al. Consumer knowledge and attitudes toward healthy eating in Croatia: a cross-sectional study. Arh Hig Rada Toksikol. 2017;68(2):153-8. https://doi.org/10.1515/aiht-2 017-68-2939.

9. Seguin RA, Aggarwal A, Vermeylen F, Drewnowski A. Consumption frequency of foods away from home linked with higher body mass index and lower fruit and vegetable intake among adults: a cross-sectional study. J Environ Public Health. 2016:2016:3074241.

10. Guthrie JF, Lin BH, Frazao E. Role of food prepared away from home in the American diet, 1977-78 versus 1994-96: changes and consequences. J Nut Educ Behav. 2002:34(3):140-50. https://doi.org/10.1016/S1499-404 6(06)60083-3.

11. Larson NI, Perry CL, Story M, Neumark-Sztainer D. Food preparation by young adults is associated with better diet quality. J Am Diet Assoc. 2006; 106(12):2001-7. https://doi.org/10.1016/j.jada.2006.09.008

12. Lachat C, Nago E, Verstraeten R, Roberfroid D, Van Camp J, Kolsteren P. Eating out of home and its association with dietary intake: a systematic review of the evidence. Obes Rev. 2012;13(4):329-46. https://doi.org/1 0.1111/j.1467-789X.2011.00953.x.

13. Orfanos P, Naska A, Trichopoulou A, Grioni S, Boer JM, van Bakel MM, et al, Eating out of home: energy, macro- and micronutrient intakes in 10 European countries. The European prospective investigation into Cancer and nutrition. Eur J Clin Nutr. 2009;63(S4):S239-62. https://doi.org/10.1038/ ejcn.2009.84.

14. Satia JA, Galanko JA, Siega-Riz AM. Eating at fast-food restaurants is associated with dietary intake, demographic, psychosocial and behavioural factors among African Americans in North Carolina. Public Health Nutr. 2004;7(8):1089-96. https://doi.org/10.1079/PHN2004662.

15. Smith KJ, McNaughton SA, Gall SL, Blizzard L, Dwyer T, Venn AJ. Takeaway food consumption and its associations with diet quality and abdominal obesity: a cross-sectional study of young adults. Int J Behav Nutr Phys Act. 2009;6(1):29. https://doi.org/10.1186/1479-5868-6-29.

16. Pereira MA, Kartashov Al, Ebbeling CB, Van Horn L, Slattery ML, Jacobs DR Jr, et al. Fast-food habits, weight gain, and insulin resistance (the CARDIA study): 15-year prospective analysis. Lancet. 2005;365(9453):36-42. https:// doi.org/10.1016/S0140-6736(04)17663-0.

17. Paeratakul S, Ferdinand DP, Champagne CM, Ryan DH, Bray GA. Fast-food consumption among US adults and children: dietary and nutrient intake profile. J Am Diet Assoc. 2003;103(10):1332-8. https://doi.org/10.1016/S0002 8223(03)01086-1.

18. Mills S, Brown H, Wrieden W, White M, Adams J. Frequency of eating home cooked meals and potential benefits for diet and health: cross-sectional analysis of a population-based cohort study. Int J Behav Nutr Phys Act. 2017:14(1):109. https://doi.org/10.1186/s12966-017-0567-y.

19. Prentice AM, Jebb SA. Fast foods, energy density and obesity: a possible mechanistic link. Obes Rev. 2003;4(4):187-94. https://doi.org/10.1046/j.1467789X.2003.00117.x.

20. Schroder H, Fito M, Covas MI. Association of fast food consumption with energy intake, diet quality, body mass index and the risk of obesity in a representative Mediterranean population. Br J Nutr. 2007;98(6):1274-80. https://doi.org/10.1017/S0007114507781436.

21. van der Horst K, Brunner TA, Siegrist M. Ready-meal consumption: associations with weight status and cooking skills. Public Health Nutr. 2011; 14(2):239-45. https://doi.org/10.1017/S1368980010002624.

22. Dominguez $\amalg$, Martinez-Gonzalez MA, Basterra-Gortari FJ, Gea A, Barbagallo M, Bes-Rastrollo M. Fast food consumption and gestational diabetes incidence in the SUN project. PLoS One. 2014;9(9):e106627. https://doi.org/1 0.1371/journal.pone.0106627.

23. Krishnan S, Coogan PF, Boggs DA, Rosenberg L, Palmer JR. Consumption of restaurant foods and incidence of type 2 diabetes in African American women. Am J Clin Nutr. 2010;91(2):465-71. https://doi.org/10.3945/ajcn.2 009.28682.

24. Zong G, Eisenberg DM, Hu FB, Sun Q. Consumption of meals prepared at home and risk of type 2 diabetes: an analysis of two prospective cohort studies. PLoS Med. 2016;13(7):e1002052. https://doi.org/10.1371/journal. pmed.1002052

25. Sanchez-Villegas A, Toledo E, de Irala J, Ruiz-Canela M, Pla-Vidal J, MartinezGonzalez MA. Fast-food and commercial baked goods consumption and the risk of depression. Public Health Nutr. 2012;15(3):424-32. https://doi. org/10.1017/\$1368980011001856.

26. Duffey KJ, Gordon-Larsen P, Jacobs DR Jr, Williams OD, Popkin BM. Differential associations of fast food and restaurant food consumption with $3-y$ change in body mass index: the coronary artery risk development in young adults study. Am J Clin Nutr. 2007:85(1):201-8. https://doi.org/10.1 093/ajcn/85.1.201.

27. Smith LP, Ng SW, Popkin BM. Trends in US home food preparation and consumption: analysis of national nutrition surveys and time use studies from 1965-1966 to 2007-2008. Nutr J. 2013;12(1):45. https://doi.org/10.11 86/1475-2891-12-45.

28. Ministry of Health, Labour and Welfare: The National Health and Nutrition Survey in Japan, 2015. 2017.

29. Ministry of Health, Labour and Welfare: The National Health and Nutrition Survey in Japan, 2016. 2017.

30. Saito A, Imai S, Htun NC, Okada E, Yoshita K, Yoshiike N, et al. The trends in total energy, macronutrients and sodium intake among Japanese: findings from the 1995-2016 National Health and nutrition survey. Br J Nutr. 2018(120):424-34

31. Leech RM, Livingstone KM, Worsley A, Timperio A, McNaughton SA. Meal frequency but not snack frequency is associated with micronutrient intakes and overall diet quality in Australian men and women. J Nutr. 2016;146(10): 2027-34. https://doi.org/10.3945/jn.116.234070.

32. Zhang L, Cordeiro LS, Liu J, Ma Y. The association between breakfast skipping and body weight, nutrient intake, and metabolic measures among participants with metabolic syndrome. Nutrients. 2017;9(4). https://doi.org/1 0.3390/nu9040384

33. Murakami K, Miyake Y, Sasaki S, Tanaka K, Arakawa M. Dietary glycemic index and glycemic load in relation to risk of overweight in Japanese children and adolescents: the Ryukyus child health study. Int J Obes. 2011; 35(7):925-36. https://doi.org/10.1038/ijo.2011.59.

34. Science and Technology Agency. Standard Tables of Food Composition in Japan, 2010: Tokyo Ministry of Education, Culture, Sports, Science and Technology; 2010

35. Ministry of Health, Labour and Welfare: Dietary Reference Intakes for Japanese (2015 Edition). 2015.

36. Okubo H, Sasaki S, Murakami K, Takahashi Y. Nutritional adequacy of four dietary patterns defined by cluster analysis in Japanese women aged 18-20 years. Asia Pac J Clin Nutr. 2010;19:555-63.

37. Kobayashi S, Asakura K, Suga H, Sasaki S. Living status and frequency of eating out-of-home foods in relation to nutritional adequacy in 4,017 
Japanese female dietetic students aged 18-20 years: a multicenter crosssectional study. J Epidemiol. 2017;27(6):287-93. https://doi.org/10.1016/j.je.2 016.07.002.

38. Kohri T, Kaba N, Itoh T, Sasaki S. Effects of the National School Lunch Program on bone growth in Japanese elementary school children. J Nutr Sci Vitaminol (Tokyo). 2016;62(5):303-9. https://doi.org/10.3177/jnsv.62.303.

39. WHO/FAO: Guidelines on Food Fortification With Micronutrients. Geneva. 2006.

40. FAO/WHO: Requirements of vitamin A, iron, folate and vitamin B12. Roma 1988.

41. Board. IoMFaN: Dietary Reference Intakes: Applications in Dietary Assessment. Washington, D.C 2000

42. Wolfson JA, Bleich SN. Is cooking at home associated with better diet quality or weight-loss intention? Public Health Nutr. 2015;18(8):1397-406. https://doi.org/10.1017/S1368980014001943.

43. Huang YH, Lin CC, Lin Cl, Lin SH. Association of eating out with bone density in Taiwan. Public Health Nutr. 2017;20(17):3151-5. https://doi.org/1 $0.1017 /$ S1368980017002105.

44. Lachat C, Khanh le NB, khan NC, dung NQ, do VA N, Roberfroid D, et al. eating out of home in Vietnamese adolescents: socioeconomic factors and dietary associations. Am J Clin Nutr. 2009;90(6):1648-55. https://doi.org/10.3 945/ajcn.2009.28371.

45. Saito A, Okada E, Tarui I, Matsumoto M, Takimoto H. The Association between Milk and Dairy Products Consumption and Nutrient Intake Adequacy among Japanese Adults: Analysis of the 2016 National Health and nutrition survey. Nutrients. 2019:11.

46. Zhang W, Iso H, Ohira T, Date OC, Tanabe N, Kikuchi S, et al. Associations of dietary iron intake with mortality from cardiovascular disease: the JACC study. J Epidemiol. 2012;22(6):484-93. https://doi.org/10.2188/jea.JE2012 0006.

47. Saito A, Matsumoto M, Hyakutake A, Saito M, Okamoto N, Tsuji M. The frequency of cooking dinner at home and its association with nutrient intake adequacy among married young-to-moderate-aged Japanese women: the POTATO study. J Nutr Sci. 2019;8:e14. https://doi.org/10.1017/ jns.2019.9.

48. McGowan L, Pot GK, Stephen AM, Lavelle F, Spence M, Raats M, et al. The influence of socio-demographic, psychological and knowledge-related variables alongside perceived cooking and food skills abilities in the prediction of diet quality in adults: a nationally representative crosssectional study. Int J Behav Nutr Phys Act. 2016;13(1):111. https://doi.org/1 0.1186/s12966-016-0440-4

49. Ministry of Health, Labour and Welfare: Health Japan 21 (the Second Term). 2012.

50. Iwaoka F, Yoshiike N, Date C, Shimada T, Tanaka H. A validation study on a method to estimate nutrient intake by family members through a household-based food-weighing survey. J Nutr Sci Vitaminol (Tokyo). 2001; 47(3):222-7. https://doi.org/10.3177/jnsv.47.222.

51. Asakura K, Sasaki S. School lunches in Japan: their contribution to healthie nutrient intake among elementary-school and junior high-school children. Public Health Nutr. 2017;20(9):1523-33. https://doi.org/10.1017/S136898001 7000374.

\section{Publisher's Note}

Springer Nature remains neutral with regard to jurisdictional claims in published maps and institutional affiliations.

Ready to submit your research? Choose BMC and benefit from:

- fast, convenient online submission

- thorough peer review by experienced researchers in your field

- rapid publication on acceptance

- support for research data, including large and complex data types

- gold Open Access which fosters wider collaboration and increased citations

- maximum visibility for your research: over $100 \mathrm{M}$ website views per year

At $\mathrm{BMC}$, research is always in progress.

Learn more biomedcentral.com/submissions 